FIAN-TD-2015-12

arXiv: 1511.01836V2 [hep-th]

\title{
BRST-BV approach to conformal fields
}

\author{
R.R. Metsaev* \\ Department of Theoretical Physics, Lebedev Physical Institute, \\ Leninsky prospect 53, Moscow 119991, Russia
}

\begin{abstract}
Using the BRST-BV approach, we consider totally symmetric arbitrary integer spin conformal fields propagating in flat space. For such fields, we obtain the ordinary-derivative BRST-BV Lagrangian that is invariant under gauge transformations. In our approach, the ordinary-derivative Lagrangian and gauge transformations are constructed in terms of the respective traceless gauge fields and traceless gauge transformation parameters. We also obtain a realization of conformal algebra symmetries on the space of fields and antifields entering the BRST-BV formulation of conformal fields.
\end{abstract}

\footnotetext{
*E-mail: metsaev@lpi.ru
} 


\section{Introduction}

The BRST approach [1] is a powerful method of modern quantum field theory. In gauge field theory, this approach turns out to be very convenient for studying ultraviolet divergences and the renormalization procedure. Nowadays, the BRST approach is the main method for finding the relativistic invariant $S$-matrix. We also note that the use of the BRST approach for studying string field theory led to an interesting new development of this approach [2]. Namely, extended versions of the BRST approach involving antifields were developed and it was demonstrated that such extended versions turn out to be not only efficient approaches for studying quantum field theory but also powerful approaches for constructing theories of classical gauge fields. The BRST approach that involves antifields [3] will be referred to as the BRST-BV approach in this paper.

For aesthetic reasons, conformal fields have attracted considerable interest during a long period of time. The duality conjecture by Maldacena also triggered interest in studying various aspects of conformal fields. In the framework of AdS/CFT correspondence, conformal fields manifest themselves as boundary values of nonnormalizable solutions of the Dirichlet problem for massless fields propagating in AdS space. Plugging a solution of the Dirichlet problem into the action of a massless AdS field gives a functional of boundary values of the massless AdS field, which is referred to as the effective action. For massless spin-2 and arbitrary integer spin- $s$ fields, it was demonstrated explicitly in the respective Ref.[4] and Ref.[5] that the ultraviolet divergence of the effective action turns out to be a respective higher-derivative action for conformal spin- 2 and for arbitrary integer spin-s fields. Interesting interrelations between partition functions of AdS fields and partition functions of conformal fields are discussed in Ref.[6]. Taking the important role played by conformal fields in the AdS/CFT correspondence into account, we think that study of these fields by using various approaches is well motivated. In this respect, we note then that the BRST-BV Lagrangian formulation of conformal fields has not been discussed previously in the literature. The major aim of this paper is to develop a BRST-BV Lagrangian formulation for totally symmetric arbitrary integer spin conformal fields 1

The BRST-BV formulation of conformal fields we develop in this paper is closely related to the ordinary-derivative metric-like formulation of conformal fields. The ordinary-derivative metric-like formulation of conformal fields in terms of double-traceless fields was developed in Refs.[7, [8]. In the BRST-BV framework, we prefer to deal with traceless fields. A formulation of conformal fields in terms of traceless fields can easily be obtained from the formulation in terms of double-traceless fields. Therefore, for the convenience of the reader, we start in Section 2 with a brief review of our ordinary-derivative metric-like formulation of conformal fields in terms of the double-traceless fields developed in Ref.[8] and then we demonstrate how such a formulation can be used to derive the ordinary-derivative metric-like formulation of conformal fields in terms of traceless fields. In Section 3, we develop a BRST-BV formulation of conformal fields. We start with the description of the field content entering our BRST-BV formulation. After that, we present our result for the ordinary-derivative BRST-BV Lagrangian of conformal fields. In our approach to conformal fields, only symmetries of the Lorentz algebra are realized manifestly. Therefore, in Section 4, we discuss a realization of conformal symmetries in the framework of our ordinary-derivative approach. Namely, we start with a brief review of a realization of the conformal algebra symmetries on the space of double-traceless fields obtained in Ref.[8] and then demonstrate how this realization can be used to derive the realization of conformal algebra symmetries on the space of traceless fields. After that, we present our result for a realization of conformal algebra

\footnotetext{
${ }^{1}$ In the literature, totally symmetric conformal fields are sometimes referred to as Fradkin-Tseytlin conformal fields.
} 
symmetries on the space of the fields and antifields entering our ordinary-derivative BRST-BV formulation of conformal fields. In Section 5, we discuss directions for future research.

\section{Ordinary-derivative metric-like formulation of conformal fields}

Field content. We start with a discussion of the metric-like formulation of conformal fields in terms of double-traceless fields developed in Ref.[8]. To streamline the presentation of the field content entering the metric-like formulation of conformal fields, we use oscillators $\alpha^{a}, \alpha^{\oplus}, \alpha^{\ominus}, \zeta$. The oscillators $\alpha^{a}$ transform as vectors under the Lorentz algebra $s o(d-1,1)$, while the oscillators $\alpha^{\oplus}, \alpha^{\ominus}, \zeta$ transform as scalars under the Lorentz algebra. Using the oscillators, we note that the fields entering the metric-like formulation of a conformal field can be collected into a ket-vector defined by

$$
|\phi\rangle=\phi\left(x, \alpha, \alpha^{\oplus}, \alpha^{\ominus}, \zeta\right)|0\rangle,
$$

where the argument $\alpha$ in (2.1) stands for the vector oscillators $\alpha^{a}$, and $x$ stands for coordinates $x^{a}$ of the $R^{d-1,1}$ space-time. We also note that the usual fields, which depend on space-time coordinates $x^{a}$, can be obtained by expanding the field $\phi(2.1)$ in the oscillators $\alpha^{a}, \alpha^{\oplus}, \alpha^{\ominus}, \zeta$. For the discussion of a spin- $s$ conformal field, we impose the following algebraic constraints on the ket-vector $|\phi\rangle(2.1)$ :

$$
\begin{gathered}
\left(N_{\alpha}+N_{\zeta}-s\right)|\phi\rangle=0, \\
\left(N_{\zeta}+N_{\alpha^{\oplus}}+N_{\alpha \ominus}-k_{s}\right)|\phi\rangle=0, \\
\left(\bar{\alpha}^{2}\right)^{2}|\phi\rangle=0, \\
k_{s} \equiv s+\frac{d-6}{2} .
\end{gathered}
$$

The definition of the operators $N_{\alpha}, N_{\alpha \oplus}, N_{\alpha}, N_{\zeta}, \bar{\alpha}^{2}$ appearing in relations (2.2)-(2.4) can be found in the Appendix (see relations (A.6)-(A.8)). From constraint (2.2), we learn that the ketvector $|\phi\rangle$ is a degree-s homogeneous polynomial in the oscillators $\alpha^{a}, \zeta$, while constraint (2.3) tells us that the ket-vector $|\phi\rangle$ is a degree- $k_{s}$ homogeneous polynomial in the oscillators $\alpha^{\oplus}, \alpha^{\ominus}, \zeta$. From (2.4) we learn that the usual tensor fields, which are obtainable by expanding the field $\phi(2.1)$ in the vector oscillators $\alpha^{a}$, are nothing but double-traceless tensor fields of the Lorentz algebra so $(d-1,1)$.

By using constraints (2.2), (2.3), we can illustrate the field content of the ket-vector $|\phi\rangle$ (2.1) explicitly. For this, we note that the general expression for $|\phi\rangle$ that satisfies algebraic constraints (2.2), (2.3) can be represented as

$$
\begin{aligned}
& |\phi\rangle \equiv \sum_{s^{\prime}=0}^{s} \frac{\zeta^{s-s^{\prime}}}{\sqrt{\left(s-s^{\prime}\right) !}}\left|\phi^{s^{\prime}}\right\rangle, \\
& \left|\phi^{s^{\prime}}\right\rangle \equiv \sum_{k^{\prime} \in\left[k_{s^{\prime}}\right]_{2}} \frac{1}{s^{\prime} !\left(\frac{k_{s^{\prime}}+k^{\prime}}{2}\right) !} \alpha^{a_{1}} \ldots \alpha^{a_{s^{\prime}}}\left(\alpha^{\ominus}\right)^{\frac{k_{s^{\prime}}+k^{\prime}}{2}}\left(\alpha^{\oplus}\right)^{\frac{k_{s^{\prime}}-k^{\prime}}{2}} \phi_{k^{\prime}}^{a_{1} \ldots a_{s^{\prime}}}|0\rangle, \\
& \left|\phi^{0}\right\rangle \equiv 0, \quad \text { for } d=4 \text {, } \\
& k_{s^{\prime}} \equiv s^{\prime}+\frac{d-6}{2},
\end{aligned}
$$


where, in (2.7) and below, the notation $k \in[n]_{2}$ implies that

$$
k=-n,-n+2, \ldots, n-2, n .
$$

In (2.7), $\phi_{k^{\prime}}^{a_{1} \ldots a_{s^{\prime}}}$ stands for a rank- $s^{\prime}$ totally symmetric tensor field of the Lorentz algebra $s o(d-$ $1,1)$. Constraint (2.4) implies that the tensor field $\phi_{k^{\prime}}^{a_{1} \ldots a_{s^{\prime}}}$ is double-traceless, $\phi_{k^{\prime}}^{a a b b a_{5} \ldots a_{s^{\prime}}}=0$, $s^{\prime} \geq 4$. We also note that the subscript $k^{\prime}$ is used to indicate conformal dimensions of fields,

$$
\Delta\left(\phi_{k^{\prime}}^{a_{1} \ldots a_{s^{\prime}}}\right)=\frac{d-2}{2}+k^{\prime} .
$$

In the case $d \geq 6$, scalar, vector, and tensor fields appearing in (2.6), (2.7) can be represented as

$$
\begin{array}{ll}
\phi_{k^{\prime}}^{a_{1} \ldots a_{s}}, & k^{\prime} \in\left[k_{s}\right]_{2} ; \\
\phi_{k^{\prime}}^{a_{1} \ldots a_{s-1}}, & k^{\prime} \in\left[k_{s}-1\right]_{2} ; \\
\ldots \ldots & \cdots \ldots \ldots . \\
\ldots \ldots & \cdots \ldots \ldots \\
\phi_{k^{\prime}}^{a}, & k^{\prime} \in\left[k_{s}-s+1\right]_{2} ; \\
\phi_{k^{\prime}}, & k^{\prime} \in\left[k_{s}-s\right]_{2} .
\end{array}
$$

We note that scalar fields are collected into the ket-vector $\left|\phi^{0}\right\rangle$. From (2.8), we see that for $d=4$, scalar fields do not enter the field content. Therefore, for $d=4$, the field content is given in (2.12)-(2.14). In other words, scalar fields $\phi_{k^{\prime}}$ enter the field content only when $d \geq 6$.

To illustrate the field content presented in (2.12)-(2.15) more explicitly, we use the shorthand notation $\phi_{k^{\prime}}^{s^{\prime}}$ for the field $\phi_{k^{\prime}}^{a_{1} \ldots a_{s^{\prime}}}$. We then note that the fields in (2.12)-(2.15) can be represented as

$$
\begin{aligned}
& \text { Field content for } d \geq 6, \quad s-\text { arbitrary integer } \\
& \begin{array}{lllllllll}
\phi_{-k_{s}}^{s} & \phi_{2-k_{s}}^{s} & \ldots & \ldots & \ldots & \ldots & \ldots & \phi_{k_{s}-2}^{s} & \phi_{k_{s}}^{s}
\end{array} \\
& \begin{array}{llllllll}
\phi_{1-k_{s}}^{s-1} & \phi_{3-k_{s}}^{s-1} & \ldots & \ldots & \ldots & \ldots & \phi_{k_{s}-3}^{s-1} & \phi_{k_{s}-1}^{s-1}
\end{array} \\
& \begin{array}{llllllll}
\ldots & \ldots & \ldots & \ldots & \ldots & \ldots & \ldots & \ldots
\end{array} \\
& \begin{array}{llllll}
\phi_{s-1-k_{s}}^{1} & \phi_{s+1-k_{s}}^{1} \quad \ldots & \ldots & \phi_{k_{s}-s-1}^{1} & \phi_{k_{s}-s+1}^{1}
\end{array} \\
& \phi_{s-k_{s}}^{0} \quad \phi_{s-k_{s}+2}^{0} \quad \ldots \quad \phi_{k_{s}-s-2}^{0} \quad \phi_{k_{s}-s}^{0}
\end{aligned}
$$

We now discuss representations for the Lagrangian in terms of double-traceless and traceless fields in turn.

Representation for the Lagrangian in terms of double-trace fields. An ordinary-derivative representation for the action and the Lagrangian of a spin- $s$ conformal field in terms of doubletraceless fields described above was obtained in Ref.[8]:

$$
S=\int d^{d} x \mathcal{L}
$$




$$
\begin{aligned}
\mathcal{L}= & \frac{1}{2}\left\langle\phi\left|\left(1-\frac{1}{4} \alpha^{2} \bar{\alpha}^{2}\right)\left(\square-M^{\oplus \oplus}\right)\right| \phi\right\rangle+\frac{1}{2}\langle\bar{L} \phi \mid \bar{L} \phi\rangle, \\
& M^{\oplus \oplus} \equiv \alpha^{\oplus} \bar{\alpha}^{\oplus}, \\
& \bar{L} \equiv \bar{\alpha} \partial-\frac{1}{2} \alpha \partial \bar{\alpha}^{2}-\Pi^{[1,2]} \bar{e}_{1}+\frac{1}{2} e_{1} \bar{\alpha}^{2}, \\
& e_{1} \equiv \zeta e_{\zeta} \bar{\alpha}^{\oplus}, \quad \bar{e}_{1} \equiv-\alpha^{\oplus} e_{\zeta} \bar{\zeta}, \quad e_{\zeta} \equiv\left(\frac{2 s+d-4-N_{\zeta}}{2 s+d-4-2 N_{\zeta}}\right)^{1 / 2}, \\
& \Pi^{[1,2]} \equiv 1-\alpha^{2} \frac{1}{2\left(2 N_{\alpha}+d\right)} \bar{\alpha}^{2} .
\end{aligned}
$$

In (2.18), the bra-vector is defined in accordance with the rule $\langle\phi| \equiv(|\phi\rangle)^{\dagger}$. We also use the conventions that $|\bar{L} \phi\rangle \equiv \bar{L}|\phi\rangle$ and $\langle\bar{L} \phi| \equiv(|\bar{L} \phi\rangle)^{\dagger}$. The remaining notation can be found in the Appendix (see relations (A.5)-(A.8) $)$.

We refer to the quantity $|\bar{L} \phi\rangle$ as the conformal de Donder divergence. We recall that the standard de Donder divergence, which enters the massless field Lagrangian in $R^{d-1,1}$, is obtained by setting $e_{1}=0, \bar{e}_{1}=0$ in (2.20) (see, e.g., Ref.[9]). From (2.18), we see that it is the use of the conformal de Donder divergence that allows us to considerably simplify the representation for the gauge invariant Lagrangian of a conformal field.2

To describe gauge symmetries of Lagrangian (2.18), we note that appropriate gauge transformation parameters can be collected into the ket-vector

$$
|\xi\rangle=\xi\left(x, \alpha, \alpha^{\oplus}, \alpha^{\ominus}, \zeta\right)|0\rangle
$$

which satisfies the algebraic constraints

$$
\begin{aligned}
& \left(N_{\alpha}+N_{\zeta}-s+1\right)|\xi\rangle=0, \\
& \left(N_{\zeta}+N_{\alpha^{\oplus}}+N_{\alpha}-k_{s}\right)|\xi\rangle=0, \\
& \bar{\alpha}^{2}|\xi\rangle=0,
\end{aligned}
$$

where $k_{s}$ is defined in (2.5). From (2.24), we learn that the ket-vector $|\xi\rangle$ is a degree- $(s-1)$ homogeneous polynomial in $\alpha^{a}, \zeta$, while from (2.25), we learn that the ket-vector $|\xi\rangle$ is a degree$k_{s}$ homogeneous polynomial in $\zeta, \alpha^{\oplus}, \alpha^{\ominus}$. Taking this into account, we see that the ket-vector $|\xi\rangle$ can be expanded in the oscillators as follows

$$
\begin{aligned}
|\xi\rangle= & \sum_{s^{\prime}=0}^{s-1} \frac{\zeta^{s-1-s^{\prime}}}{\sqrt{\left(s-1-s^{\prime}\right) !}}\left|\xi^{s^{\prime}}\right\rangle \\
& \left|\xi^{s^{\prime}}\right\rangle \equiv \sum_{k^{\prime} \in\left[k_{s^{\prime}}+1\right]_{2}} \frac{1}{s^{\prime} !\left(\frac{k_{s^{\prime}}+1+k^{\prime}}{2}\right) !} \alpha^{a_{1}} \ldots \alpha^{a_{s^{\prime}}}\left(\alpha^{\ominus}\right)^{\frac{k_{s^{\prime}+1+k^{\prime}}}{2}}\left(\alpha^{\oplus}\right)^{\frac{k_{s^{\prime}}+1-k^{\prime}}{2}} \xi_{k^{\prime}-1}^{a_{1} \ldots a_{s^{\prime}}}|0\rangle,
\end{aligned}
$$

where $k_{s^{\prime}}$ is defined in (2.9). In (2.28), the gauge transformation parameter $\xi_{k^{\prime}}^{a_{1} \ldots a_{s^{\prime}}}$ is a rank- $s^{\prime}$ totally symmetric tensor field of the Lorentz algebra $s o(d-1,1)$. Constraint (2.26) tells us that

\footnotetext{
2 The use of modified de Donder divergences for studying the metric-like formulation of fields in AdS space can be found in Refs.[10, 11]. For a massive field in $R^{d-1,1}$, the modified de Donder divergence was found in Ref.[12].
} 
the gauge transformation parameter $\xi_{k^{\prime}}^{a_{1} \ldots a_{s^{\prime}}}$ is traceless, $\xi_{k^{\prime}}^{a a a_{3} \ldots a_{s^{\prime}}}=0, s^{\prime} \geq 2$. The conformal dimension of the gauge transformation parameter $\xi_{k^{\prime}}^{a_{1} \ldots a_{s^{\prime}}}$ is given by

$$
\Delta\left(\xi_{k^{\prime}}^{a_{1} \ldots a_{s^{\prime}}}\right)=\frac{d-2}{2}+k^{\prime}
$$

Using ket-vectors $|\phi\rangle$ (2.6) and $|\xi\rangle$ (2.27), gauge transformations can be written as

$$
\delta|\phi\rangle=G|\xi\rangle, \quad G \equiv \alpha \partial-e_{1}-\alpha^{2} \frac{1}{2 N_{\alpha}+d-2} \bar{e}_{1}
$$

where the operators $e_{1}, \bar{e}_{1}$ are defined in (2.21).

Representation for the Lagrangian in terms of traceless fields. A Lagrangian formulation of a conformal field in terms of traceless fields can easily be obtained by using the formulation in terms of double-traceless fields discussed above. For this, we note that the double-traceless ket-vector $|\phi\rangle$ (2.1), (2.4) can be decomposed into two traceless ket-vectors, denoted by $\left|\phi_{I}\right\rangle,\left|\phi_{I I}\right\rangle$, as

$$
|\phi\rangle=\left|\phi_{I}\right\rangle+\alpha^{2} \mathcal{N}\left|\phi_{I I}\right\rangle, \quad \mathcal{N} \equiv\left(\left(2 N_{\alpha}+d\right)\left(2 N_{\alpha}+d-2\right)\right)^{-1 / 2},
$$

where the tracelessness constraint for the ket-vectors $\left|\phi_{I}\right\rangle,\left|\phi_{I I}\right\rangle$ takes the form

$$
\bar{\alpha}^{2}\left|\phi_{I}\right\rangle=0, \quad \bar{\alpha}^{2}\left|\phi_{I I}\right\rangle=0
$$

Plugging (2.31) in (2.18), we obtain a representation for the Lagrangian in terms of traceless ketvectors,

$$
\mathcal{L}=\frac{1}{2}\left\langle\phi_{I}\left|\left(\square-M^{\oplus \oplus}\right)\right| \phi_{I}\right\rangle-\frac{1}{2}\left\langle\phi_{I I}\left|\left(\square-M^{\oplus \oplus}\right)\right| \phi_{I I}\right\rangle+\frac{1}{2}\langle\bar{L} \phi \mid \bar{L} \phi\rangle,
$$

where the operator $M^{\oplus \oplus}$ takes the same form as in (2.19). The de Donder divergence $|\bar{L} \phi\rangle$ appearing in (2.33) is represented in terms of the traceless ket-vectors $\left|\phi_{I}\right\rangle,\left|\phi_{I I}\right\rangle$ as

$$
\begin{aligned}
& \bar{L}|\phi\rangle= \bar{L}_{I}\left|\phi_{I}\right\rangle+L_{I I}\left|\phi_{I I}\right\rangle, \\
& \bar{L}_{I} \equiv \bar{\alpha} \partial-\bar{e}_{1} \\
& L_{I I} \equiv-A^{a} \partial^{a} f_{1 \zeta}+e_{1} f_{1 \zeta}^{-1}, \\
& f_{1 \zeta} \equiv\left(\frac{2 s+d-6-2 N_{\zeta}}{2 s+d-4-2 N_{\zeta}}\right)^{1 / 2}, \\
& A^{a} \equiv \alpha^{a}-\alpha^{2} \frac{1}{2 N_{\alpha}+d} \bar{\alpha}^{a},
\end{aligned}
$$

where $e_{1}, \bar{e}_{1}$ are given in (2.21). In terms of the traceless ket-vectors $\left.\left|\phi_{I}\right\rangle, \phi_{I I}\right\rangle$, gauge transformations (2.30) can be represented as

$$
\begin{aligned}
\delta\left|\phi_{I}\right\rangle= & G_{I}|\xi\rangle, \\
\delta\left|\phi_{I I}\right\rangle= & \bar{G}_{I I}|\xi\rangle \\
& G_{I} \equiv A^{a} \partial^{a}-e_{1}, \\
& \bar{G}_{I I} \equiv f_{1 \zeta} \bar{\alpha} \partial-f_{1 \zeta}^{-1} \bar{e}_{1},
\end{aligned}
$$


where the gauge transformation parameter $|\xi\rangle$ takes the same form as in (2.27). Gauge transformations (2.39), (2.40) can easily be obtained from the ones in (2.30) by noticing that the inverse to relation in (2.31) takes the form

$$
\begin{aligned}
& \left|\phi_{I}\right\rangle=\Pi^{[1,2]}|\phi\rangle, \\
& \left|\phi_{I I}\right\rangle=\frac{1}{2}\left(\frac{2 N_{\alpha}+d-2}{2 N_{\alpha}+d}\right)^{1 / 2} \bar{\alpha}^{2}|\phi\rangle,
\end{aligned}
$$

where the operator $\Pi^{[1,2]}$ is defined in (2.22).

From the presentation given above, we see that our formulations in terms of double-traceless and traceless fields are completely equivalent.

\section{BRST-BV Lagrangian of conformal fields}

Field content. In order to streamline the presentation of the field content entering our BRST-BV formulation of a conformal field, we introduce a Grassmann coordinate $\theta, \theta^{2}=0$, Grassmann-even oscillators $\alpha^{a}, \alpha^{\oplus}, \alpha^{\ominus}, \zeta$, and Grassmann-odd oscillators $\eta, \rho$. The Grassmann-even oscillators $\alpha^{a}$ transform as vectors under the Lorentz algebra $s o(d-1,1)$, while the Grassmann coordinate $\theta$, the Grassmann-even oscillators $\alpha^{\oplus}, \alpha^{\ominus}, \zeta$, and the Grassmann-odd oscillators $\eta, \rho$ transform as scalars under the Lorentz algebra. Using the Grassmann coordinate $\theta$ and the oscillators, the fields entering our BRST-BV formulation of a conformal field can be collected into a ket-vector defined by

$$
|\Phi\rangle=\Phi\left(x, \theta, \alpha, \alpha^{\oplus}, \alpha^{\ominus}, \zeta, \eta, \rho\right)|0\rangle,
$$

where the argument $\alpha$ in (3.1) stands for the vector oscillators $\alpha^{a}$, while the argument $x$ stands for coordinates $x^{a}$ of the $R^{d-1,1}$ space-time. By definition, the field $\Phi$ in (3.1) is considered to be Grassmann even. We also note that usual fields, which depend on the space-time coordinates $x^{a}$, can be obtained by expanding the field $\Phi\left(\underline{3.1}\right.$ ) in the $\theta$ and the above-introduced oscillators $\alpha^{a}$, $\alpha^{\oplus}, \alpha^{\ominus}, \zeta, \eta, \rho$.

For the discussion of a spin-s conformal field, we impose the following algebraic constraints on the ket-vector $|\Phi\rangle(\underline{3.1})$ :

$$
\begin{aligned}
& \left(N_{\alpha}+N_{\zeta}+N_{\eta}+N_{\rho}-s\right)|\Phi\rangle=0, \\
& \left(N_{\zeta}+N_{\alpha^{\oplus}}+N_{\alpha \ominus}-k_{s}\right)|\Phi\rangle=0, \\
& \bar{\alpha}^{2}|\Phi\rangle=0,
\end{aligned}
$$

where $k_{s}$ is defined in (2.5), while the operators $N_{\alpha}, N_{\alpha^{\oplus}}, N_{\alpha \ominus}, N_{\zeta}, N_{\eta}, N_{\rho}, \bar{\alpha}^{2}$ appearing in (3.2)-(3.4) are defined in the Appendix (see relations (A.6)-A.8). From constraint (3.2), we learn that the ket-vector $|\Phi\rangle$ is a degree-s homogeneous polynomial in the oscillators $\alpha^{a}, \zeta, \eta, \rho$, while constraint (3.3) tells us that the ket-vector $|\Phi\rangle$ is a degree- $k_{s}$ homogeneous polynomial in the oscillators $\alpha^{\oplus}, \alpha^{\ominus}, \zeta$. From constraint (3.4) we learn that the usual tensor fields, which are obtainable by expanding the field $\Phi(\underline{3.1})$ in the vector oscillators $\alpha^{a}$, are nothing but traceless tensor fields of the Lorentz algebra $s o(d-1,1)$.

The use of constraints (3.2), (3.3) allows us to illustrate a catalogue of scalar, vector, and tensor fields entering the ket-vector $|\Phi\rangle($ (3.1) in a rather straightforward way. For this, we note that the 
expansion of $|\Phi\rangle(3.1)$ in the Grassmann coordinate $\theta$ and the Grassmann-odd oscillators $\eta, \rho$ can be presented as

$$
\begin{aligned}
|\Phi\rangle= & |\phi\rangle+\theta\left|\phi_{*}\right\rangle, \\
& |\phi\rangle=\left|\phi_{I}\right\rangle+\rho|c\rangle+\eta|\bar{c}\rangle+\rho \eta\left|\phi_{I I}\right\rangle, \\
& \left|\phi_{*}\right\rangle=\left|\phi_{* I}\right\rangle+\rho\left|\bar{c}_{*}\right\rangle+\eta\left|c_{*}\right\rangle+\rho \eta\left|\phi_{* I I}\right\rangle .
\end{aligned}
$$

We now note that the ket-vectors appearing in the right-hand side of (3.6), (3.7) depend only on the vector oscillators $\alpha^{a}$ and the scalar oscillators $\zeta, \alpha^{\oplus}, \alpha^{\ominus}$. In this paper, the ket-vectors $|\phi\rangle$ (3.6) and $\left|\phi_{*}\right\rangle$ (3.7) are referred to as the respective ket-vectors of fields and antifields 3 We note that the algebraic constraints for the ket-vector of fields $|\phi\rangle$ and the ket-vector of antifields $\left|\phi_{*}\right\rangle$ take the same form as the ones for the $|\Phi\rangle$ in (3.2)-(3.4). This implies that expansions of the ket-vector of fields and the ket-vector of antifields in terms of the respective scalar, vector, and tensor fields and antifields of the Lorentz algebra $s o(d-1,1)$ take the same form. Therefore, for illustration purpose, we consider an expansion of the ket-vector of fields $|\phi\rangle$. Taking constraints (3.2), (3.3) into account, we verify that the ket-vector $\left|\phi_{I, I I}\right\rangle,|c\rangle,|\bar{c}\rangle$ (3.6) depends on the oscillators $\alpha^{a}, \alpha^{\oplus}$, $\alpha^{\ominus}, \zeta$ as

$$
\begin{aligned}
& \left|\phi_{I}\right\rangle=\sum_{s^{\prime}=0}^{s} \frac{\zeta^{s-s^{\prime}}}{\sqrt{\left(s-s^{\prime}\right) !}}\left|\phi_{I}^{s^{\prime}}\right\rangle \\
& \left|\phi_{I}^{s^{\prime}}\right\rangle \equiv \sum_{k^{\prime} \in\left[k_{s^{\prime}}\right]_{2}} \frac{1}{s^{\prime} !\left(\frac{k_{s^{\prime}}+k^{\prime}}{2}\right) !} \alpha^{a_{1}} \ldots \alpha^{a_{s^{\prime}}}\left(\alpha^{\ominus}\right)^{\frac{k_{s^{\prime}}+k^{\prime}}{2}}\left(\alpha^{\oplus}\right)^{\frac{k_{s^{\prime}}-k^{\prime}}{2}} \phi_{I, k^{\prime}}^{a_{1} \ldots a_{s^{\prime}}}|0\rangle, \\
& |c\rangle=\sum_{s^{\prime}=0}^{s-1} \frac{\zeta^{s-1-s^{\prime}}}{\sqrt{\left(s-1-s^{\prime}\right) !}}\left|c^{s^{\prime}}\right\rangle, \\
& \left|c^{s^{\prime}}\right\rangle \equiv \sum_{k^{\prime} \in\left[k_{s^{\prime}}+1\right]_{2}} \frac{1}{s^{\prime} !\left(\frac{k_{s^{\prime}}+1+k^{\prime}}{2}\right) !} \alpha^{a_{1}} \ldots \alpha^{a_{s^{\prime}}}\left(\alpha^{\ominus}\right)^{\frac{k_{s^{\prime}}+1+k^{\prime}}{2}}\left(\alpha^{\oplus}\right)^{\frac{k_{s^{\prime}}+1-k^{\prime}}{2}} c_{k^{\prime}}^{a_{1} \ldots a_{s^{\prime}}}|0\rangle, \\
& |\bar{c}\rangle=\sum_{s^{\prime}=0}^{s-1} \frac{\zeta^{s-1-s^{\prime}}}{\sqrt{\left(s-1-s^{\prime}\right) !}}\left|\bar{c}^{s^{\prime}}\right\rangle \\
& \left|\bar{c}^{s^{\prime}}\right\rangle \equiv \sum_{k^{\prime} \in\left[k_{s^{\prime}}+1\right]_{2}} \frac{1}{s^{\prime} !\left(\frac{k_{s^{\prime}}+1+k^{\prime}}{2}\right) !} \alpha^{a_{1}} \ldots \alpha^{a_{s^{\prime}}}\left(\alpha^{\ominus}\right)^{\frac{k_{s^{\prime}}+1+k^{\prime}}{2}}\left(\alpha^{\oplus}\right)^{\frac{k_{s^{\prime}}+1-k^{\prime}}{2}} \bar{c}_{k^{\prime}}^{a_{1} \ldots a_{s^{\prime}}}|0\rangle, \\
& \left|\phi_{I I}\right\rangle=\sum_{s^{\prime}=0}^{s-2} \frac{\zeta^{s-2-s^{\prime}}}{\sqrt{\left(s-2-s^{\prime}\right) !}}\left|\phi_{I I}^{s^{\prime}}\right\rangle \\
& \left|\phi_{I I}^{s^{\prime}}\right\rangle \equiv \sum_{k^{\prime} \in\left[k_{s^{\prime}}+2\right]_{2}} \frac{1}{s^{\prime} !\left(\frac{k_{s^{\prime}}+2+k^{\prime}}{2}\right) !} \alpha^{a_{1}} \ldots \alpha^{a_{s^{\prime}}}\left(\alpha^{\ominus}\right)^{\frac{k_{s^{\prime}}+2+k^{\prime}}{2}}\left(\alpha^{\oplus}\right)^{\frac{k_{s^{\prime}}+2-k^{\prime}}{2}} \phi_{I I, k^{\prime}}^{a_{1} \ldots a_{s^{\prime}}}|0\rangle,
\end{aligned}
$$

\footnotetext{
${ }^{3}$ Our terminology for ket-vectors in (3.6), (3.7) does not coincide with the standard terminology used in the literature. Matching our simplified terminology and the standard one is discussed at the end of the Appendix.
} 
where, in (3.8), $\left|\phi_{I}^{0}\right\rangle=0$ if $d=4$. The $k_{s^{\prime}}$ appearing in (3.8)-(3.11) is defined in (2.9).

Relations (3.8)-(3.11) explicitly illustrate the field content entering the ket-vector of fields (3.6). We see a set of fields denoted by $\phi_{I, k^{\prime}}^{a_{1} \ldots a_{s^{\prime}}}, c_{k^{\prime}}^{a_{1} \ldots a_{s^{\prime}}}, \bar{c}_{k^{\prime}}^{a_{1} \ldots a_{s^{\prime}}}, \phi_{I I, k^{\prime}}^{a_{1} \ldots a_{s^{\prime}}}$. Fields with the values $s^{\prime}=$ $0, s^{\prime}=1$, and $s^{\prime} \geq 2$ correspond to the respective scalar, vector, and tensor fields of the Lorentz algebra $s o(d-1,1)$. All the tensor fields are totally symmetric tensor fields. From constraint (3.4), we learn that all the tensor fields are realized as traceless tensors of the Lorentz algebra $s o(d-1,1)$. As is well known, in the BRST-BV approach, each field enters the game with a corresponding antifield. This implies that in our approach, a catalogue of the antifields is described by the antifields denoted by $\phi_{* I, k^{\prime}}^{a_{1} \ldots a_{s^{\prime}}}, c_{* k^{\prime}}^{a_{1} \ldots a_{s^{\prime}}}, \bar{c}_{* k^{\prime}}^{a_{1} \ldots a_{s^{\prime}}}, \phi_{* I I, k^{\prime}}^{a_{1} \ldots a_{s^{\prime}}}$. We also note that ket-vectors of antifields $\left|\phi_{* I}\right\rangle,\left|c_{*}\right\rangle,\left|\bar{c}_{*}\right\rangle,\left|\phi_{* I I}\right\rangle$ given in (3.7) are expressed in terms of the antifields $\phi_{* I, k^{\prime}}^{a_{1} \ldots a_{s^{\prime}}}$, $c_{* k^{\prime}}^{a_{1} \ldots a_{s^{\prime}}}, \bar{c}_{* k^{\prime}}^{a_{1} \ldots a_{s^{\prime}}}, \phi_{* I I, k^{\prime}}^{a_{1} \ldots a_{s^{\prime}}}$ in the same way as in (3.8)-(3.11). Thus, the ket-vector $|\Phi\rangle$ describes fields and antifields that are realized as scalars, vectors, and totally symmetric traceless tensors of the Lorentz algebra $s o(d-1,1)$.

BRST-BV Lagrangian of conformal fields. A general representation for the BRST-BV action that describes the dynamics of fields and antifields in the $R^{d-1,1}$ space-time takes the form [2]

$$
\begin{aligned}
& S=\int d^{d} x \mathcal{L}, \\
& \mathcal{L}=\frac{1}{2} \int d \theta\langle\Phi|Q| \Phi\rangle .
\end{aligned}
$$

The BRST operator $Q$ appearing in expression (3.13) for the Lagrangian admits the general representation

$$
Q=\theta\left(\square-M^{\oplus \oplus}\right)+M^{\eta a} \partial^{a}+M^{\oplus \eta}+M^{\eta \eta} \partial_{\theta},
$$

where the notation $\partial_{\theta}$ is used for the left derivative of the Grassmann coordinate, $\partial_{\theta}=\partial / \partial \theta$, while the notation $\square=\partial^{a} \partial^{a}$ is used for the D'Alembert operator in the space-time $R^{d-1,1}$.

From the expression for the BRST operator given in (3.14), we see that the BRST operator is defined by the operators $M^{\oplus \oplus}, M^{\eta a}, M^{\oplus \eta}, M^{\eta \eta}$. In what follows, the operator $M^{\oplus \oplus}$ is referred to as the mass operator, while the operators $M^{\eta a}, M^{\oplus \eta}, M^{\eta \eta}$ are referred to as spin operators. The mass and spin operators depend only on the oscillators, i.e., the mass and spin operators do not depend on the space-time coordinates $x^{a}$, on the Grassmann coordinate $\theta$, and on the derivatives $\partial^{a}, \partial_{\theta}$.

The basic equation for the BRST operator $Q^{2}=0$ amounts to the following (anti)commutation relations for the mass and spin operators:

$$
\begin{array}{ll}
\left\{M^{\eta a}, M^{\eta b}\right\}=-2 \eta^{a b} M^{\eta \eta}, & \\
\left\{M^{\oplus \eta}, M^{\oplus \eta}\right\}=2 M^{\oplus \oplus} M^{\eta \eta}, & \\
{\left[M^{\oplus \oplus}, M^{\eta a}\right]=0, \quad\left[M^{\oplus \oplus}, M^{\oplus \eta}\right]=0,} & {\left[M^{\oplus \oplus}, M^{\eta \eta}\right]=0,} \\
\left\{M^{\eta a}, M^{\oplus \eta}\right\}=0, \quad\left[M^{\eta a}, M^{\eta \eta}\right]=0, & {\left[M^{\oplus \eta}, M^{\eta \eta}\right]=0 .}
\end{array}
$$

BRST-BV action (3.12) is invariant under the gauge transformations given by

$$
\delta|\Phi\rangle=Q|\Xi\rangle
$$


where $|\Xi\rangle$ stands for a ket-vector of the gauge transformation parameters. The ket-vector $|\Xi\rangle$ can be represented as

$$
|\Xi\rangle=\Xi\left(x, \theta, \alpha, \alpha^{\oplus}, \alpha^{\ominus}, \zeta, \eta, \rho\right)|0\rangle,
$$

where the arguments $x$ and $\alpha$ in (3.20) stand for the respective coordinates $x^{a}$ of the space-time $R^{d-1,1}$ and vector oscillators $\alpha^{a}$. Usual gauge transformation parameters, which depend on the space-time coordinates $x^{a}$, are obtained by expanding the generating function $\Xi$ (3.20) in the $\theta$ and the oscillators $\alpha^{a}, \alpha^{\oplus}, \alpha^{\ominus}, \zeta, \eta, \rho$. The generating function $\Xi$ is considered to be Grassmann odd. The ket-vector $|\Xi\rangle$ satisfies, by definition, the algebraic constraints

$$
\begin{aligned}
& \left(N_{\alpha}+N_{\zeta}+N_{\eta}+N_{\rho}-s\right)|\Xi\rangle=0, \\
& \left(N_{\zeta}+N_{\alpha^{\oplus}}+N_{\alpha}-k_{s}\right)|\Xi\rangle=0, \\
& \bar{\alpha}^{2}|\Xi\rangle=0,
\end{aligned}
$$

where $k_{s}$ is defined in (2.5).

Comparing the algebraic constraints given in (3.2)-(3.4) and (3.21)-(3.23), we see that ketvectors $|\Phi\rangle$ and $|\Xi\rangle$ satisfy the same constraints. Therefore, using the results of our analysis of the constraints for the ket-vector of gauge fields $|\Phi\rangle$, we conclude the following:

i) The ket-vector of gauge transformation parameters $|\Xi\rangle$ is built of gauge transformation parameters that are realized as scalar, vector, and totally symmetric traceless tensor fields of the Lorentz algebra $s o(d-1,1)$.

ii) An expansion of the ket-vector of the gauge transformation parameter $|\Xi\rangle$ in terms of scalar, vector, and tensor gauge transformation parameters is obtained simply by replacing the gauge fields in relations (3.5) - (3.11) by the scalar, vector and tensor gauge transformation parameters.

It is clear from the discussion given above that all that is required to find the BRST-BV action and the corresponding gauge transformations is the BRST operator. We also see that in order to build the BRST operator, we should find a realization for the mass and spin operators on the space of ket-vector $|\Phi\rangle$ and plug that realization for the mass and spin operators into expression for the BRST operator in (3.14). In our approach, a spin- $s$ conformal field is described by the ket-vector $|\Phi\rangle$ that by definition, satisfies constraints (3.2)-(3.4). This implies that on space of the ket-vector $|\Phi\rangle$ subject to algebraic constraints (3.2)-(3.4), we should find a realization of (anti)commutation relations for the mass and spin operators (3.15) $-(3.18)$. Our result for the mass and spin operators is as follows 4

$$
\begin{aligned}
& M^{\oplus \oplus}=\alpha^{\oplus} \bar{\alpha}^{\oplus}, \\
& M^{\eta a}=\eta g_{\rho \zeta} \bar{\alpha}^{a}+A^{a} \bar{g}_{\eta \zeta} \bar{\eta}, \\
& M^{\eta \eta}=\eta \bar{\eta}, \\
& M^{\oplus \eta}=\eta l_{\rho \zeta}^{\oplus} \bar{\zeta}+\zeta \bar{l}_{\eta \zeta}^{\oplus} \bar{\eta},
\end{aligned}
$$

where we use the following notation

$$
A^{a} \equiv \alpha^{a}-\alpha^{2} \frac{1}{2 N_{\alpha}+d} \bar{\alpha}^{a}
$$

${ }^{4}$ We note that restrictions imposed by (anti)commutation relations (3.15)-3.18) do not allow determining the mass and spin operators uniquely. To fix the mass and spin operators uniquely, one has to also analyse the restrictions imposed by conformal algebra symmetries. Such an analysis can be done by using the method we described in the framework of the metric-like approach to conformal fields in Appendix B in Ref.[8]. A discussion of conformal symmetries in our BRST-BV approach can be found in Sec. 4 in this paper. 


$$
\begin{gathered}
g_{\rho \zeta} \equiv\left(\frac{2 s+d-4-2 N_{\zeta}-2 N_{\rho}}{2 s+d-4-2 N_{\zeta}}\right)^{1 / 2}, \\
\bar{g}_{\eta \zeta} \equiv-\left(\frac{2 s+d-4-2 N_{\zeta}-2 N_{\eta}}{2 s+d-4-2 N_{\zeta}}\right)^{1 / 2}, \\
l_{\rho \zeta}^{\oplus} \equiv \alpha^{\oplus} e_{\zeta}\left(\frac{2 s+d-4-2 N_{\zeta}-2 N_{\rho}}{2 s+d-4-2 N_{\zeta}}\right)^{-1 / 2}, \\
\bar{l}_{\eta \zeta}^{\oplus} \equiv \bar{\alpha}^{\oplus} e_{\zeta}\left(\frac{2 s+d-4-2 N_{\zeta}-2 N_{\eta}}{2 s+d-4-2 N_{\zeta}}\right)^{-1 / 2}, \\
e_{\zeta} \equiv\left(\frac{2 s+d-4-N_{\zeta}}{2 s+d-4-2 N_{\zeta}}\right)^{1 / 2},
\end{gathered}
$$

while the definition of the operators $N_{\alpha}, N_{\alpha \oplus}, N_{\alpha} \ominus, N_{\zeta}$ can be found in the Appendix (see relations (A.7), (A.8).

The following remarks are in order.

i) From (3.14), we see that our BRST operator is a degree-2 polynomial in the space-time derivatives $\partial^{a}$. In other words, there are no higher-derivative terms in our BRST-BV Lagrangian formulation of conformal fields. We note then that it is the field content described in (3.8)-(3.11) that allows us to develop the ordinary-derivative BRST-BV Lagrangian formulation of conformal fields.

ii) We demonstrate how our BRST-BV Lagrangian is related to the Lagrangian entering the metriclike formulation in terms of traceless fields (2.33). For this, we equate all fields and antifields with a nonzero ghost number to zero:

$$
|c\rangle=0, \quad|\bar{c}\rangle=0, \quad\left|\phi_{* I}\right\rangle=0, \quad\left|c_{*}\right\rangle=0, \quad\left|\phi_{*_{I I}}\right\rangle=0 .
$$

The definition of the ghost number and the values of ghost numbers for fields and antifields can be found in the Appendix (see relations (A.15), (A.16)). Using relations (3.34), we find that BRSTBV Lagrangian (3.13) takes the form

$$
\mathcal{L}=\frac{1}{2}\left\langle\phi_{I}\left|\left(\square-M^{\oplus \oplus}\right)\right| \phi_{I}\right\rangle-\frac{1}{2}\left\langle\phi_{I I}\left|\left(\square-M^{\oplus \oplus}\right)\right| \phi_{I I}\right\rangle-\left\langle\bar{c}_{*}\left|\bar{L}_{I}\right| \phi_{I}\right\rangle-\left\langle\bar{c}_{*}\left|L_{I I}\right| \phi_{I I}\right\rangle-\frac{1}{2}\left\langle\bar{c}_{*}|| \bar{c}_{*}\right\rangle,
$$

where the operators $\bar{L}_{I}, L_{I I}$ appearing in (3.35) are defined in (2.35)-2.38). The equation of motion for the antifield $\left|\bar{c}_{*}\right\rangle$ obtained from Lagrangian (3.35) allows us to express the antifield $\left|\bar{c}_{*}\right\rangle$ in terms of the fields $\left|\phi_{I}\right\rangle,\left|\phi_{I I}\right\rangle$ as

$$
\left.-\left|\bar{c}_{*}\right\rangle=\bar{L}_{I}\left|\phi_{I}\right\rangle+\left|L_{I I}\right| \phi_{I I}\right\rangle .
$$

Plugging (3.36) in (3.35), we find that Lagrangian (3.35) takes the form of the Lagrangian in the metric-like approach given in (2.33), (2.34).

iii) Using the Siegel gauge $\left|\phi_{*}\right\rangle=0$, we verify that Lagrangian (3.13) leads to the following simple expression for gauge-fixed Lagrangian:

$$
\mathcal{L}=\frac{1}{2}\left\langle\phi_{I}\left|\left(\square-M^{\oplus \oplus}\right)\right| \phi_{I}\right\rangle-\frac{1}{2}\left\langle\phi_{I I}\left|\left(\square-M^{\oplus \oplus}\right)\right| \phi_{I I}\right\rangle+\left\langle\bar{c}\left|\left(\square-M^{\oplus \oplus}\right)\right| c\right\rangle .
$$

We note that when passing from the relation for a gauge invariant Lagrangian in (3.13) to the gaugefixed Lagrangian in (3.37), we changed the sign of the Faddeev-Popov antighost field, $|\bar{c}\rangle \rightarrow-|\bar{c}\rangle$. 
Gauge-fixed Lagrangian (3.37) is invariant under the global BRST and anti-BRST symmetries given by

$$
\begin{array}{lll}
\mathrm{s}\left|\phi_{I, I I}\right\rangle=G_{I, I I}|c\rangle, & \mathbf{s}|c\rangle=0, & \mathbf{s}|\bar{c}\rangle=\bar{L}_{I}\left|\phi_{I}\right\rangle+L_{I I}\left|\phi_{I I}\right\rangle, \\
\overline{\mathbf{s}}\left|\phi_{I, I I}\right\rangle=G_{I, I I}|\bar{c}\rangle, & \overline{\mathbf{s}}|c\rangle=-\bar{L}_{I}\left|\phi_{I}\right\rangle-L_{I I}\left|\phi_{I I}\right\rangle, & \overline{\mathbf{s}}|\bar{c}\rangle=0,
\end{array}
$$

where the operators $\bar{L}_{I}, L_{I I}$, and $G_{I, I I}$, are respectively given in (2.35), (2.36), and (2.41), (2.42). We also note that the global BRST and anti-BRST transformations presented in (3.38), (3.39) satisfy the nilpotence relations $s^{2}=0, \bar{s}^{2}=0$ for arbitrary Faddeev-Popov fields, while the nilpotence relation $s \bar{s}+\bar{s} s=0$ is respected only for on-shell Faddeev-Popov fields $|\bar{c}\rangle,|c\rangle$.

\section{Realization of conformal symmetries}

Conformal symmetries of fields propagating in space-time $R^{d-1,1}$ are described by the conformal algebra $s o(d, 2)$. Note however that only symmetries of the Lorentz algebra $s o(d-1,1)$ are manifest in the framework of our approach. Therefore in order to complete our description of conformal fields we should provide a realization of symmetries of the conformal algebra on space of fields and antifields entering the BRST-BV action. Because symmetries of the Lorentz algebra $s o(d-1,1)$ are realized manifestly in the framework of our approach, we represent the $s o(d, 2)$ algebra in the basis of the Lorentz algebra $s o(d-1,1)$. In basis of the Lorentz algebra, the generators of the conformal algebra $s o(d, 2)$ are described by the Poincaré translation generators $P^{a}$, dilatation generator $D$, conformal boosts generators $K^{a}$, and generators of the Lorentz algebra $s o(d-1,1)$ denoted by $J^{a b}$. We assume the following normalization for commutation relations of the $\operatorname{so}(d, 2)$ algebra generators

$$
\begin{array}{ll}
{\left[D, P^{a}\right]=-P^{a},} & {\left[P^{a}, J^{b c}\right]=\eta^{a b} P^{c}-\eta^{a c} P^{b},} \\
{\left[D, K^{a}\right]=K^{a},} & {\left[K^{a}, J^{b c}\right]=\eta^{a b} K^{c}-\eta^{a c} K^{b}} \\
{\left[P^{a}, K^{b}\right]=\eta^{a b} D-J^{a b},} & {\left[J^{a b}, J^{c e}\right]=\eta^{b c} J^{a e}+3 \text { terms. }}
\end{array}
$$

A general representation for the generators of the $s o(d, 2)$ algebra on space of conformal fields is given by the following relations

$$
\begin{aligned}
& P^{a}=\partial^{a}, \\
& J^{a b}=x^{a} \partial^{b}-x^{b} \partial^{a}+M^{a b}, \\
& D=x^{a} \partial^{a}+\Delta, \\
& K^{a}=-\frac{1}{2} x^{b} x^{b} \partial^{a}+x^{a} D+M^{a b} x^{b}+R^{a} .
\end{aligned}
$$

In (4.4), the $\Delta$ stands for operator of conformal dimension, while, in (4.3), (4.5), the $M^{a b}$ stands for spin operator of the Lorentz algebra $s o(d-1,1)$. The spin operator satisfies the commutation relations

$$
\left[M^{a b}, M^{c e}\right]=\eta^{b c} M^{a e}+3 \text { terms. }
$$

For totally symmetric fields considered in this paper, the spin operator $M^{a b}$ takes the following well known form

$$
M^{a b}=\alpha^{a} \bar{\alpha}^{b}-\alpha^{b} \bar{\alpha}^{a}
$$


Operator $R^{a}$ appearing in (4.5) does not depend on coordinates $x^{a}$ of space-time $R^{d-1,1}$ and, in general, depends on the derivatives $\partial^{a}$. We note also that, in the standard higher-derivative approach to conformal fields, the operator $R^{a}$ is often equal to zero, while in the ordinary-derivative approach this operator turns out to be nontrivial. It is the finding of the operator $R^{a}$ that provides the real difficulty in the problem of realization of the conformal boost symmetries in the framework of the ordinary-derivative approach.

In this section, we present our result for a realization of the operators $\Delta$ and $R^{a}$ on space of the fields and antifields entering our ordinary-derivative BRST-BV formulation of conformal fields. For the reader convenience, we start with the description of the operators $\Delta$ and $R^{a}$ for the metriclike double-traceless fields [8] and the metric-like traceless fields we discussed in Sec,2] in this paper.

Operators $\Delta$ and $R^{a}$ for metric-like double-traceless fields. Expression for the conformal dimension operator can easily be read from relation in (2.11). Namely, using (2.11) and (2.6), we see that realization of the operator $\Delta$ on space of the double-traceless ket-vector $|\phi\rangle(2.6)$ takes the form

$$
\Delta=M^{\ominus \oplus}+\frac{d-2}{2}, \quad M^{\ominus \oplus} \equiv N_{\alpha} \ominus N_{\alpha} \oplus .
$$

Realization of the operator $R^{a}$ on space of the double-traceless ket-vector (2.6) is given by [8]

$$
R^{a}=-\frac{1}{2} M^{\ominus \ominus} \partial^{a}+M^{\ominus a}
$$

where we use the notation

$$
\begin{aligned}
M^{\ominus \ominus} \equiv & 4 \alpha^{\ominus} \bar{\alpha}^{\ominus}, \\
M^{\ominus a} \equiv & r_{0,1} \bar{\alpha}^{a}+\widetilde{A}^{a} \bar{r}_{0,1}, \\
& \widetilde{A}^{a} \equiv \alpha^{a}-\alpha^{2} \frac{1}{2 N_{\alpha}+d-2} \bar{\alpha}^{a}, \\
& r_{0,1} \equiv 2 \zeta e_{\zeta} \bar{\alpha}^{\ominus}, \quad \bar{r}_{0,1} \equiv-2 \alpha^{\ominus} e_{\zeta} \bar{\zeta}, \quad e_{\zeta} \equiv\left(\frac{2 s+d-4-N_{\zeta}}{2 s+d-4-2 N_{\zeta}}\right)^{1 / 2} .
\end{aligned}
$$

The operator $\widetilde{A}^{a}$ appears in (4.11) because it is this operator that respects double-tracelessness constraint (2.4). Namely, if a ket-vector $|\phi\rangle$ satisfies double-tracelessness constraint (2.4), then the following relation holds true: $\left(\bar{\alpha}^{2}\right)^{2} \widetilde{A}^{a}|\phi\rangle=0$.

Operators $\Delta$ and $R^{a}$ for metric-like traceless fields. Realization of the operators $\Delta$ and $R^{a}$ on space of the traceless ket-vectors $\left|\phi_{I}\right\rangle,\left|\phi_{I I}\right\rangle$ can easily be obtained by using the realization of $\Delta$ and $R^{a}$ on space of the double-traceless ket-vector above-discussed. To this end we use the interrelations between the double-traceless ket-vector $|\phi\rangle$ and the traceless ket-vectors $\left|\phi_{I}\right\rangle,\left|\phi_{I I}\right\rangle$ given in (2.31) and (2.43), (2.44). Doing so, we see immediately that a realization of the operator $\Delta$ on space of the ket-vectors $\left|\phi_{I}\right\rangle,\left|\phi_{I I}\right\rangle$ takes the same form as before

$$
\Delta=M^{\ominus \oplus}+\frac{d-2}{2}, \quad M^{\ominus \oplus} \equiv N_{\alpha}-N_{\alpha \oplus} .
$$

For the operator $R^{a}$, we get the following representation

$$
R^{a}=-\frac{1}{2} M^{\ominus \ominus} \partial^{a}+M^{\ominus a}
$$


where the realization of the operator $M^{\ominus \ominus}$ on space of the ket-vectors $\left.\left|\phi_{I}\right\rangle, \phi_{I I}\right\rangle$ takes the form

$$
M^{\ominus \ominus}=4 \alpha^{\ominus} \bar{\alpha}^{\ominus},
$$

while the realization of the operator $M^{\ominus a}$ on space of the ket-vectors $\left.\left|\phi_{I}\right\rangle, \phi_{I I}\right\rangle$ is given by

$$
\begin{aligned}
M^{\ominus a}\left|\phi_{I}\right\rangle & =\left(r_{0,1} \bar{\alpha}^{a}+A^{a} \bar{r}_{0,1}\right)\left|\phi_{I}\right\rangle+2 r_{0,1} A^{a} \mathcal{N}\left|\phi_{I I}\right\rangle, \\
M^{\ominus a}\left|\phi_{I I}\right\rangle & =\left(r_{0,1} u_{11 \zeta} \bar{\alpha}^{a}+A^{a} u_{11 \zeta} \bar{r}_{0,1}\right)\left|\phi_{I I}\right\rangle-2 \mathcal{N} \bar{r}_{0,1} \bar{\alpha}^{a}\left|\phi_{I}\right\rangle,
\end{aligned}
$$

and, in relations (4.17), (4.18), we use the following notation:

$$
\begin{aligned}
& A^{a} \equiv \alpha^{a}-\alpha^{2} \frac{1}{2 N_{\alpha}+d} \bar{\alpha}^{a} \\
& r_{0,1} \equiv 2 \zeta e_{\zeta} \bar{\alpha}^{\ominus}, \quad \bar{r}_{0,1} \equiv-2 \alpha^{\ominus} e_{\zeta} \bar{\zeta}, \quad e_{\zeta} \equiv\left(\frac{2 s+d-4-N_{\zeta}}{2 s+d-4-2 N_{\zeta}}\right)^{1 / 2}, \\
& u_{11 \zeta} \equiv\left(\frac{\left(2 s+d-4-2 N_{\zeta}\right)\left(2 s+d-8-2 N_{\zeta}\right)}{\left(2 s+d-6-2 N_{\zeta}\right)^{2}}\right)^{1 / 2}, \\
& \mathcal{N} \equiv\left(\left(2 N_{\alpha}+d\right)\left(2 N_{\alpha}+d-2\right)\right)^{-1 / 2} .
\end{aligned}
$$

We note that the operator $A^{a}$ (4.19) appears in relations (4.17), (4.18) because it is this operator that respects the tracelessness constraints (2.32). Namely, taking into account that the ket-vectors $\left|\phi_{I}\right\rangle,\left|\phi_{I I}\right\rangle$ satisfy tracelessness constraints (2.32), we verify that the following relations hold true: $\bar{\alpha}^{2} A^{a}\left|\phi_{I, I I}\right\rangle=0$.

Operators $\Delta$ and $R^{a}$ for BRST-BV fields and antifields. We now describe our main result in this section. In our BRST-BV approach, fields and antifields are described by the ket-vector (3.1) subject to algebraic constraints (3.2)-(3.4). We find the following realization of the conformal dimension operator $\Delta$ on space of ket-vector $|\Phi\rangle$ (3.1):

$$
\begin{aligned}
\Delta= & 2 \theta \partial_{\theta}+M^{\eta \rho}+M^{\ominus \oplus}+\frac{d-2}{2}, \\
& M^{\ominus \oplus} \equiv N_{\alpha}-N_{\alpha^{\oplus}} \\
& M^{\eta \rho} \equiv N_{\eta}-N_{\rho} .
\end{aligned}
$$

Comparing operator $\Delta$ (4.23) with the one for double-traceless and traceless fields in (4.8), (4.14), we note that, in our BRST-BV approach, the conformal dimension operator $\Delta$ (4.23) depends not only on the operator $M^{\ominus \oplus}$ but also on the Grassmann coordinate $\theta$, the Grassmann derivative $\partial_{\theta}$ and the Grassmann odd oscillators entering the operators $N_{\eta}, N_{\rho}$ in (4.25). Note also that, the operator $\Delta$ in (4.23), when restricted to the space of ket-vectors $\left|\phi_{I}\right\rangle,\left|\phi_{I I}\right\rangle$ (3.6) entering BRSTBV ket-vector (3.6), turns out to be equal to the one in metric-like approach (4.14), as it should be.

For the operator $R^{a}$, we find the following realization on space of ket-vector $|\Phi\rangle($ 3.1):

$$
R^{a}=-\frac{1}{2} M^{\ominus \ominus} \partial^{a}+M^{\ominus a}+2 \theta M^{\rho a},
$$

where operators $M^{\ominus \ominus}, M^{\ominus a}, M^{\rho a}$ appearing in (4.26) are defined by the following relations:

$$
M^{\ominus \ominus}=4 \alpha^{\ominus} \bar{\alpha}^{\ominus},
$$




$$
\begin{aligned}
M^{\rho a}= & \rho f_{\eta \zeta} \bar{\alpha}^{a}+A^{a} \bar{f}_{\rho \zeta} \bar{\rho} \\
& f_{\rho \zeta} \equiv\left(\frac{2 s+d-4-2 N_{\zeta}-2 N_{\rho}}{2 s+d-4-2 N_{\zeta}}\right)^{1 / 2} \\
& \bar{f}_{\eta \zeta} \equiv\left(\frac{2 s+d-4-2 N_{\zeta}-2 N_{\eta}}{2 s+d-4-2 N_{\zeta}}\right)^{1 / 2}, \\
M^{\ominus a}= & A^{\zeta} \bar{\alpha}^{a}+A^{a} \bar{A}^{\zeta}, \\
& A^{a} \equiv \alpha^{a}-\alpha^{2} \frac{1}{2 N_{\alpha}+d} \bar{\alpha}^{a}, \\
& A^{\zeta} \equiv \zeta \bar{e}_{\eta \rho \zeta}^{\ominus}+h_{\zeta}^{\ominus} \rho \eta \bar{\zeta}, \\
& \bar{A}^{\zeta} \equiv e_{\eta \rho \zeta}^{\ominus} \bar{\zeta}+\zeta \bar{\eta} \bar{\rho} \bar{h}_{\zeta}^{\ominus},
\end{aligned}
$$

and, in relations (4.33), (4.34), we use the following notation

$$
\begin{aligned}
& \bar{e}_{\eta \rho \zeta}^{\ominus} \equiv 2 e_{\zeta} u_{\eta \rho \zeta} \bar{\alpha}^{\ominus}, \quad e_{\eta \rho \zeta}^{\ominus} \equiv-2 e_{\zeta} u_{\eta \rho \zeta} \alpha^{\ominus}, \quad e_{\zeta} \equiv\left(\frac{2 s+d-4-N_{\zeta}}{2 s+d-4-2 N_{\zeta}}\right)^{1 / 2}, \\
& u_{\eta \rho \zeta} \equiv\left(\frac{\left(2 s+d-4-2 N_{\zeta}\right)\left(2 s+d-4-2 N_{\zeta}-2 N_{\eta}-2 N_{\rho}\right)}{\left(2 s+d-4-2 N_{\zeta}-2 N_{\eta}\right)\left(2 s+d-4-2 N_{\zeta}-2 N_{\rho}\right)}\right)^{1 / 2}, \\
& h_{\zeta}^{\ominus} \equiv 4 e_{\zeta}\left(\left(2 s+d-4-2 N_{\zeta}\right)\left(2 s+d-6-2 N_{\zeta}\right)\right)^{-1 / 2} \alpha^{\ominus}, \\
& \bar{h}_{\zeta}^{\ominus} \equiv-4 e_{\zeta}\left(\left(2 s+d-4-2 N_{\zeta}\right)\left(2 s+d-6-2 N_{\zeta}\right)\right)^{-1 / 2} \bar{\alpha}^{\ominus} .
\end{aligned}
$$

From relations in (4.27)-(4.38), we see that the operators $M^{\ominus \ominus}, M^{\ominus a}, M^{\rho a}$ depend only on the oscillators. These relations provide the complete description of the operator $R^{a}$.

Using relations for the operators $M^{a b}, \Delta$, and $R^{a}$, we verify that BRST-BV action (3.12) is invariant under transformation of the conformal algebra symmetries $s o(d, 2)$ given in (4.2)-(4.5). Using expressions for $\Delta$ and $R^{a}$ above described, we also verify the commutation relation for conformal boost generators, $\left[K^{a}, K^{b}\right]=0$.

Superalgebra $\operatorname{ssp}(d-1,1 \mid 2)$. Some subset of the spin operators involved in our BRST-BV formulation of conformal field turns out to be related to the superalgebra $\operatorname{ssp}(d-1,1 \mid 2)$. In order to explain what just has been said let us consider the spin operators $M^{\eta a}, M^{\eta \eta}$, which enter the BRST operator (3.14). Also let us consider the spin operators $M^{\eta \rho}, M^{\rho a}, M^{a b}$ which enter generators of the conformal symmetries. We now observe that, if to the spin operators $M^{\eta a}, M^{\eta \eta}$ entering the BRST operator and the spin operators $M^{\eta \rho}, M^{\rho a}, M^{a b}$, entering the conformal symmetries, we add a new spin operator $M^{\rho \rho}$, which we define by the following expression $M^{\rho \rho}=\rho \bar{\rho}$, then we get the superalgebra $\operatorname{osp}(d-1,1 \mid 2)$. Namely, we checked that the just mentioned spin operators indeed satisfy the commutation relations of the superalgebra $\operatorname{csp}(d-1,1 \mid 2)$. For the reader convenience, we note that we use the following normalization for commutation relations of the superalgebra $\operatorname{osp}(d-1,1 \mid 2)$ :

$$
\begin{aligned}
& {\left[M^{a b}, M^{c e}\right]=\eta^{b c} M^{a e}+3 \text { terms }} \\
& {\left[M^{\eta \rho}, M^{\eta \eta}\right]=2 M^{\eta \eta}}
\end{aligned}
$$




$$
\begin{aligned}
& {\left[M^{\eta \rho}, M^{\rho \rho}\right]=-2 M^{\rho \rho},} \\
& {\left[M^{\eta \eta}, M^{\rho \rho}\right]=M^{\eta \rho},} \\
& {\left[M^{\eta \rho}, M^{\eta a}\right]=M^{\eta a},} \\
& {\left[M^{\eta \rho}, M^{\rho a}\right]=-M^{\rho a},} \\
& {\left[M^{\eta \eta}, M^{\rho a}\right]=M^{\eta a},} \\
& {\left[M^{\rho \rho}, M^{\eta a}\right]=M^{\rho a},} \\
& {\left[M^{\eta a}, M^{b c}\right]=\eta^{a b} M^{\eta c}-\eta^{a c} M^{\eta b},} \\
& {\left[M^{\rho a}, M^{b c}\right]=\eta^{a b} M^{\rho c}-\eta^{a c} M^{\rho b},} \\
& \left\{M^{\rho a}, M^{\eta b}\right\}=\eta^{a b} M^{\eta \rho}+M^{a b}, \\
& \left\{M^{\eta a}, M^{\eta b}\right\}=-2 \eta^{a b} M^{\eta \eta}, \\
& \left\{M^{\rho a}, M^{\rho b}\right\}=2 \eta^{a b} M^{\rho \rho} .
\end{aligned}
$$

We recall also the superalgebra $\operatorname{osp}(d-1,1 \mid 2)$ can be decomposed as

$$
\underbrace{M^{a b}}_{s o(d-1,1)}, \underbrace{M^{\eta \rho}, M^{\eta \eta}, M^{\rho \rho}}_{s p(2)}, \underbrace{M^{\eta a}, M^{\rho a}}_{\text {coset }} .
$$

\section{Conclusions}

To summarize, in this paper, we developed a BRST-BV Lagrangian formulation of arbitrary integer spin totally symmetric conformal fields propagating in flat space. So far, the Lagrangian BRST$\mathrm{BV}$ formulation of arbitrary integer spin conformal fields was not discussed in the literature 5 We note, however, that in the earlier literature, the BRST approach has been extensively used for studying nonconformal (massless and massive) fields propagating in flat and AdS spaces (see, e.g., Refs.[16]-[22]). We think that the use of methods developed in Refs.[16]-[22] might be helpful for better understanding various aspects of the BRST formulation of conformal fields. In this paper, we studied totally symmetric conformal fields. In Refs. [23, 24, 25], mixed-symmetry conformal fields were studied in the framework of a metric-like approach. We expect that applications of the BRST approach to the mixed-symmetry conformal fields might provide a simpler setup for studying the mixed-symmetry conformal fields. We note that in this paper, we dealt with free conformal fields. In the BRST approach framework, many interesting methods have been developed for studying interacting nonconformal (massless and massive) fields propagating in flat and AdS spaces (see, e.g., Refs.[26]-[28]). The application of those methods for studying conformal fields should lead to better understanding the interacting conformal fields. Needless to say, the use of the BRST-BV approach for studying long conformal fields propagating in flat space [29, 30] and short conformal

\footnotetext{
${ }^{5}$ A higher-derivative Lagrangian for arbitrary integer spin conformal fields invariant under global BRST transformations was discussed in Ref.[13]. In the framework of the BRST-BV approach, equations of motion for arbitrary integer spin conformal fields were discussed in Ref.[14]. In the framework of the BRST-BV Lagrangian approach, study of a spin-2 conformal field (Weyl gravity) can be found in Ref.[15].
} 
fields propagating in the AdS space [31] seems to be fruitful directions to pursue. Application of BRST approach to the study of algebraic aspects of higher-spin algebras along the lines of [32] could also be of some interest.

Acknowledgments. We thank A. Semikhatov for useful comments. This work was supported by the RFBR Grant No.14-02-01172.

\section{Appendix A Notation and conventions}

We use mostly positive flat metric $\eta^{a b}=(-,+, \ldots,+)$. In scalar products, we drop the flat metric, $X^{a} Y^{a} \equiv \eta_{a b} X^{a} Y^{b}$. Vector indices of the Lorentz algebra $s o(d-1,1)$ run over $a, b, c, e=$ $0,1, \ldots, d-1$.

Coordinates in space-time $R^{d-1,1}$ and Grassmann coordinate are denoted by $x^{a}$ and $\theta$ respectively. Derivatives of the space-time coordinates $x^{a}$ and left derivative of the Grassmann coordinate $\theta$ are denoted by $\partial^{a} \equiv \eta^{a b} \partial / \partial x^{b}$ and $\partial_{\theta}$ respectively. The normalization for the integral over $\theta$ is as follows $\int d \theta \theta=1$. Hermitian conjugation rules for the coordinates and the derivatives are defined as

$$
\left(x^{a}, \theta\right)^{\dagger}=\left(x^{a}, \theta\right), \quad\left(\partial^{a}, \partial_{\theta}\right)^{\dagger}=\left(-\partial^{a}, \partial_{\theta}\right) .
$$

For product of two operators $A, B$ having arbitrary ghost numbers, hermitian conjugation is defined according to the rule $(A B)^{\dagger}=B^{\dagger} A^{\dagger}$.

(Anti)commutation relations for creation operators $\alpha^{a}, \alpha^{\oplus}, \alpha^{\ominus}, \zeta, \eta, \rho$ and the respective annihilation operators $\bar{\alpha}^{a}, \bar{\alpha}^{\ominus}, \bar{\alpha}^{\oplus}, \bar{\zeta}, \bar{\rho}, \bar{\eta}$, the vacuum $|0\rangle$ and the hermitian conjugation rules are defined as

$$
\begin{aligned}
& {\left[\bar{\alpha}^{a}, \alpha^{b}\right]=\eta^{a b}, \quad\left[\bar{\alpha}^{\oplus}, \alpha^{\ominus}\right]=1, \quad\left[\bar{\alpha}^{\ominus}, \alpha^{\oplus}\right]=1, \quad[\bar{\zeta}, \zeta]=1,} \\
& \{\bar{\rho}, \eta\}=1, \quad\{\bar{\eta}, \rho\}=1 \text {, } \\
& \bar{\alpha}^{a}|0\rangle=0, \quad \bar{\alpha}^{\oplus}|0\rangle=0, \quad \bar{\alpha}^{\ominus}|0\rangle=0, \quad \bar{\zeta}|0\rangle=0, \\
& \bar{\eta}|0\rangle=0, \quad \bar{\rho}|0\rangle=0, \\
& \alpha^{a \dagger}=\bar{\alpha}^{a}, \quad \alpha^{\oplus \dagger}=\bar{\alpha}^{\oplus}, \quad \alpha^{\ominus \dagger}=\bar{\alpha}^{\ominus}, \quad \zeta^{\dagger}=\bar{\zeta}, \\
& \eta^{\dagger}=\bar{\eta}, \quad \rho^{\dagger}=\bar{\rho} \text {. }
\end{aligned}
$$

The creation and annihilation operators are referred to as oscillators in this paper. The oscillators $\alpha^{a}, \bar{\alpha}^{a}$ transform as vectors of the Lorentz algebra $s o(d-1,1)$, while the oscillators $\alpha^{\oplus}, \bar{\alpha}^{\ominus}$, $\alpha^{\ominus}, \bar{\alpha}^{\oplus}, \zeta, \bar{\zeta}, \eta, \bar{\rho}, \rho, \bar{\eta}$ transform as scalars of the Lorentz algebra $s o(d-1,1)$.

Throughout this paper the following notation for the products of the derivatives and the oscillators is used

$$
\begin{array}{llll}
\square \equiv \partial^{a} \partial^{a}, & & \\
\alpha \partial \equiv \alpha^{a} \partial^{a}, & \bar{\alpha} \partial \equiv \bar{\alpha}^{a} \partial^{a}, & \alpha^{2} \equiv \alpha^{a} \alpha^{a}, & \bar{\alpha}^{2} \equiv \bar{\alpha}^{a} \bar{\alpha}^{a}, \\
N_{\alpha} \equiv \alpha^{a} \bar{\alpha}^{a}, & N_{\alpha^{\oplus}} \equiv \alpha^{\oplus} \bar{\alpha}^{\ominus}, & N_{\alpha} \equiv \alpha^{\ominus} \bar{\alpha}^{\oplus}, & N_{\zeta} \equiv \zeta \bar{\zeta}, \\
N_{\eta} \equiv \eta \bar{\rho}, & N_{\rho} \equiv \rho \bar{\eta} . &
\end{array}
$$


Using (A.4), we note the following hermitian conjugation rules for operators in (A.7), (A.8)

$$
\begin{array}{lll}
N_{\alpha}^{\dagger}=N_{\alpha}, & N_{\alpha^{\oplus}}^{\dagger}=N_{\alpha \ominus}, \quad N_{\alpha \ominus}^{\dagger}=N_{\alpha^{\oplus}}, \quad N_{\zeta}^{\dagger}=N_{\zeta}, \\
N_{\eta}^{\dagger}=N_{\rho}, & N_{\rho}^{\dagger}=N_{\eta} . &
\end{array}
$$

On space of ket-vector $|\Phi\rangle\left(\underline{3.1}\right.$, the internal Faddeev-Popov ghost operator $N_{F P}^{\text {int }}$ is realized as

$$
N_{F P}^{\text {int }}=\theta \partial_{\theta}+N_{\eta}-N_{\rho}
$$

Using the notation $X$ for oscillator, we find the ghost number $q$ of the oscillator $X$ according to the relation $\left[N_{F P}^{\text {int }}, X\right]=q X$. Using this relation, we get the following ghost numbers of $\theta, \partial_{\theta}$, and the oscillators:

$$
\begin{array}{ll}
\operatorname{gh}\left(\alpha^{a}, \alpha^{\oplus}, \alpha^{\ominus}, \zeta\right)=0, & \operatorname{gh}\left(\bar{\alpha}^{a}, \bar{\alpha}^{\oplus}, \bar{\alpha}^{\ominus}, \bar{\zeta}\right)=0, \\
\operatorname{gh}(\theta, \eta, \bar{\rho})=1, & \operatorname{gh}\left(\partial_{\theta}, \bar{\eta}, \rho\right)=-1 .
\end{array}
$$

For ket-vectors given in (3.6), (3.7), the ghost numbers are defined as eigenvalues of the external Faddeev-Popov operator. Using the notation $\mathbf{N}_{F P}^{\text {ext }}$ for the external Faddeev-Popov operator, we note that eigenvalues of $\mathbf{N}_{F P}^{\text {ext }}$ are obtained by using the following relation:

$$
\left(N_{F P}^{\mathrm{int}}+\mathbf{N}_{F P}^{\mathrm{ext}}\right)|\Phi\rangle=0
$$

where the internal Faddeev-Popov operator $N_{F P}^{\text {int }}$ is defined in (A.11). Using (A.14), the ghost numbers of ket-vectors given in (3.6), (3.7) are found to be

$$
\begin{aligned}
& \operatorname{gh}\left(\left|\phi_{I}\right\rangle\right)=0, \quad \operatorname{gh}(|c\rangle)=1, \quad \operatorname{gh}(|\bar{c}\rangle)=-1, \quad \operatorname{gh}\left(\left|\phi_{I I}\right\rangle\right)=0, \\
& \operatorname{gh}\left(\left|\phi_{I *}\right\rangle\right)=-1, \quad \operatorname{gh}\left(\left|c_{*}\right\rangle\right)=-2, \quad \operatorname{gh}\left(\left|\bar{c}_{*}\right\rangle\right)=0, \quad \operatorname{gh}\left(\left|\phi_{I I *}\right\rangle\right)=-1 .
\end{aligned}
$$

The ghost numbers of the vacuum $|0\rangle$ is equal to zero. Taking this into account and using (A.12), we conclude that ghost numbers (A.15) of ket-vectors (3.6) coincide with ghost numbers of tensor fields appearing on the right-hand side in relations (3.8)-(3.11). The same coincidence holds true for ghost numbers (A.16) of ket-vectors (3.7) and the corresponding ghost numbers of scalar, vector, and tensor antifields which are obtainable by expanding antifields ket-vectors into the oscillators $\alpha^{a}, \alpha^{\oplus}, \alpha^{\ominus}, \zeta$.

Ghost numbers of the gauge transformation parameters can be obtained by using the following relation $\left(N_{F P}^{\text {int }}+\mathbf{N}_{F P}^{\text {ext }}+1\right)|\Xi\rangle=0$.

Throughout this paper we use the following hermitian conjugation rules for bra-vectors and ket-vectors:

$$
\begin{array}{lll}
\langle\Phi|=| \Phi\rangle^{\dagger}, & \\
\left\langle\phi_{I, I I}|=| \phi_{I, I I}\right\rangle^{\dagger}, & \langle c|=| c\rangle^{\dagger}, & \langle\bar{c}|=-| \bar{c}\rangle^{\dagger}, \\
\left\langle\phi_{* I, I I}|=-| \phi_{* I, I I}\right\rangle^{\dagger}, & \left\langle c_{*}|=-| c_{*}\right\rangle^{\dagger}, & \left\langle\bar{c}_{*}|=| \bar{c}_{*}\right\rangle^{\dagger} .
\end{array}
$$

Matching of terminology for ket-vectors in this paper and the standard terminology. For the reader convenience, we now match terminology for ket-vectors (3.6), (3.7) we use in this paper and the standard terminology used for those ket-vectors in the literature. We recall that, in this 
paper, all ket-vectors in (3.6) are referred to as fields, while all ket-vectors in (3.7) are referred to as antifields. We note that such terminology does not coincide with the standard terminology in the literature. According to the standard terminology, ket-vectors in (3.6), (3.7) having zero, positive, and negative ghost numbers are referred to as fields, ghost, and antifields respectively. Taking into account relations (A.15), A.16), this implies that, according to the standard terminology, the ketvectors $\left|\phi_{I}\right\rangle,\left|\phi_{I I}\right\rangle,\left|\bar{c}_{*}\right\rangle$ in (3.6), (3.7) are referred to as fields, the ket-vector $|c\rangle$ in (3.6) is referred to as ghost, while the ket-vectors $|\bar{c}\rangle,\left|\phi_{* I}\right\rangle,\left|c_{*}\right\rangle,\left|\phi_{*_{I I}}\right\rangle$ in (3.6), (3.7) are referred to as antifields.

\section{References}

[1] C. Becchi, A. Rouet and R. Stora, Phys. Lett. B 52, 344 (1974).

I. V. Tyutin, "Gauge Invariance in Field Theory and Statistical Physics in Operator Formalism," Lebedev Inst. preprint, No 39 (1975) [arXiv:0812.0580 [hep-th]].

[2] W. Siegel, Phys. Lett. B 149, 157 (1984) [Phys. Lett. B 151, 391 (1985)].

W. Siegel, Phys. Lett. B 149, 162 (1984) [Phys. Lett. 151B, 396 (1985)].

H. Hata, K. Itoh, T. Kugo, H. Kunitomo and K. Ogawa, Phys. Lett. B 172, 186 (1986).

H. Hata, K. Itoh, T. Kugo, H. Kunitomo and K. Ogawa, Phys. Rev. D 34, 2360 (1986).

A. Neveu and P. C. West, Phys. Lett. B 168, 192 (1986)

[3] I. A. Batalin and G. A. Vilkovisky, Phys. Lett. B 102, 27 (1981).

I. A. Batalin and G. A. Vilkovisky, Phys. Rev. D 28, 2567 (1983) [Phys. Rev. D 30, 508 (1984)].

[4] H. Liu and A. A. Tseytlin, Nucl. Phys. B 533, 88 (1998) [arXiv:hep-th/9804083].

[5] R. R. Metsaev, Phys. Rev. D 81, 106002 (2010) [arXiv:0907.4678 [hep-th]].

[6] A. A. Tseytlin, Nucl. Phys. B 877, 598 (2013) [arXiv:1309.0785 [hep-th]].

[7] R. R. Metsaev, JHEP 1201, 064 (2012) [arXiv:0707.4437[hep-th]].

[8] R. R. Metsaev, JHEP 1206, 062 (2012) [arXiv:0709.4392 [hep-th]].

[9] S. Guttenberg and G. Savvidy, SIGMA 4, 061 (2008) [arXiv:0804.0522 [hep-th]].

[10] R. R. Metsaev, Phys. Lett. B 671, 128 (2009) [arXiv:0808.3945 [hep-th]].

[11] R. R. Metsaev, Phys. Lett. B 682, 455 (2010) [arXiv:0907.2207 [hep-th]].

[12] R. R. Metsaev, Phys. Rev. D 78, 106010 (2008) [arXiv:0805.3472 [hep-th]].

[13] R. R. Metsaev, Theor. Math. Phys. 181, no. 3, 1548 (2014) [arXiv:1407.2601 [hep-th]].

[14] X. Bekaert and M. Grigoriev, J. Phys. A 46, 214008 (2013) [arXiv:1207.3439 [hep-th]].

X. Bekaert and M. Grigoriev, Nucl. Phys. B 876, 667 (2013) [arXiv:1305.0162 [hep-th]].

[15] N. Boulanger and M. Henneaux, Annalen Phys. 10, 935 (2001) [hep-th/0106065].

[16] A. K. H. Bengtsson, Nucl. Phys. B 333, 407 (1990).

A. K. H. Bengtsson, Class. Quant. Grav. 5, 437 (1988).

[17] I.L.Buchbinder, A.Pashnev, M.Tsulaia, Phys. Lett. B 523, 338 (2001) [arXiv:hep-th/0109067].

I. L. Buchbinder, V. A. Krykhtin, P. M. Lavrov, Nucl. Phys. B 762, 344 (2007) hep-th/0608005

I. L. Buchbinder and V. A. Krykhtin, Nucl. Phys. B 727, 537 (2005) [hep-th/0505092].

[18] A. Sagnotti and M. Tsulaia, Nucl. Phys. B 682, 83 (2004) [hep-th/0311257].

[19] O. A. Gelfond and M. A. Vasiliev, "Unfolding Versus BRST and Currents in Sp(2M) Invariant HigherSpin Theory," arXiv:1001.2585 [hep-th].

[20] K. Alkalaev and M. Grigoriev, Nucl. Phys. B 853, 663 (2011) [arXiv:1105.6111 [hep-th]].

K. B. Alkalaev and M. Grigoriev, Nucl. Phys. B 835, 197 (2010) [arXiv:0910.2690[hep-th]].

[21] I. L. Buchbinder and A. Reshetnyak, Nucl. Phys. B 862, 270 (2012) [arXiv:1110.5044 [hep-th]].

A. A. Reshetnyak, Phys. Part. Nucl. 41, 976 (2010) [arXiv:1002.0124 [hep-th]].

C.Burdik and A.Reshetnyak, J. Phys. Conf. Ser. 343, 012102 (2012) [arXiv:1111.5516 [hep-th]].

I. L. Buchbinder and K. Koutrolikos, JHEP 1512, 106 (2015) [arXiv:1510.06569 [hep-th]].

[22] R. R. Metsaev, "BRST-BV approach to massless fields adapted to AdS/CFT correspondence," arXiv: 1508.07928 [hep-th].

[23] M. A. Vasiliev, Nucl. Phys. B 829, 176 (2010) [arXiv:0909.5226 [hep-th]]. 
[24] R. Marnelius, "Lagrangian conformal higher spin theory," arXiv:0805.4686 [hep-th].

[25] R. R. Metsaev, J. Phys. A 43, 115401 (2010) [arXiv:0812.2861 [hep-th]].

[26] X. Bekaert, N. Boulanger and S. Cnockaert, J. Math. Phys. 46, 012303 (2005) [hep-th/0407102].

N. Boulanger and S. Cnockaert, JHEP 0403, 031 (2004) [hep-th/0402180].

[27] R. R. Metsaev, Phys. Lett. B 720, 237 (2013) [arXiv:1205.3131 [hep-th]].

[28] P. Dempster and M. Tsulaia, Nucl. Phys. B 865, 353 (2012) [arXiv:1203.5597[hep-th]].

M.Henneaux, G.L.Gomez and R.Rahman, JHEP 1208, 093 (2012) [arXiv:1206.1048 [hep-th]]. M.Henneaux, G.L.Gomez and R.Rahman, JHEP 1401, 087 (2014) [arXiv:1310.5152 [hep-th]]. I.Buchbinder, P.Dempster, M.Tsulaia, Nucl.Phys.B 877, 260 (2013) [arXiv:1308.5539[hep-th]]. I.L.Buchbinder and V.A.Krykhtin, Eur. Phys. J. C 75, no. 9, 454 (2015) [arXiv:1507.03723 [hep-th]]. M. Taronna, JHEP 1204, 029 (2012) [arXiv:1107.5843 [hep-th]].

D. Polyakov, Phys. Rev. D 93, no. 4, 045001 (2016) [arXiv:1511.04563 [hep-th]].

[29] R. R. Metsaev, JHEP 1501, 077 (2015) [arXiv:1410.7314 [hep-th]].

[30] R. R. Metsaev, JHEP 1510, 110 (2015) [arXiv:1507.06584 [hep-th]].

[31] R. R. Metsaev, Nucl. Phys. B 885, 734 (2014) [arXiv:1404.3712 [hep-th]].

[32] E. Joung and K. Mkrtchyan, JHEP 1405, 103 (2014) [arXiv:1401.7977[hep-th]].

E. Joung and K. Mkrtchyan, JHEP 1601, 003 (2016) [arXiv:1508.07332 [hep-th]]. 